
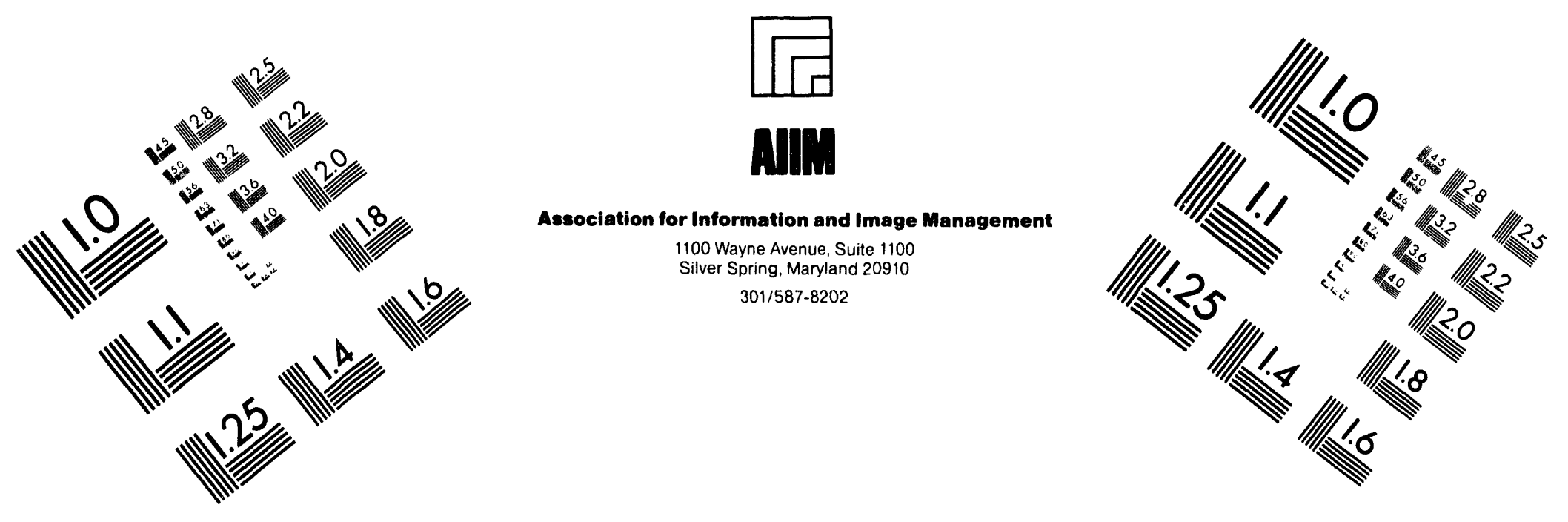

\title{
Centimeter
}

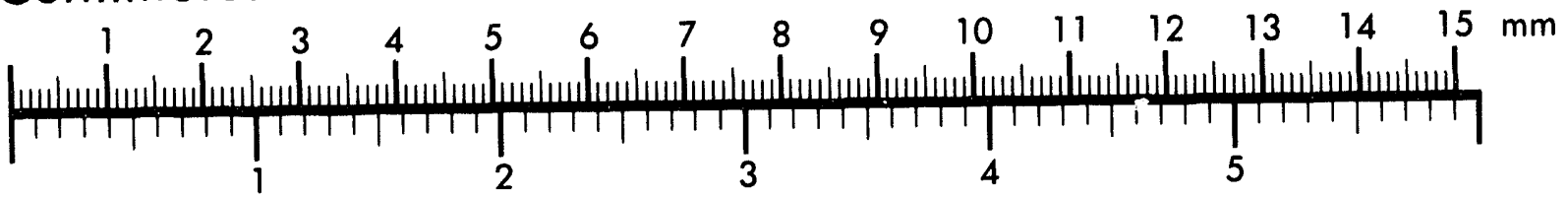
Inches
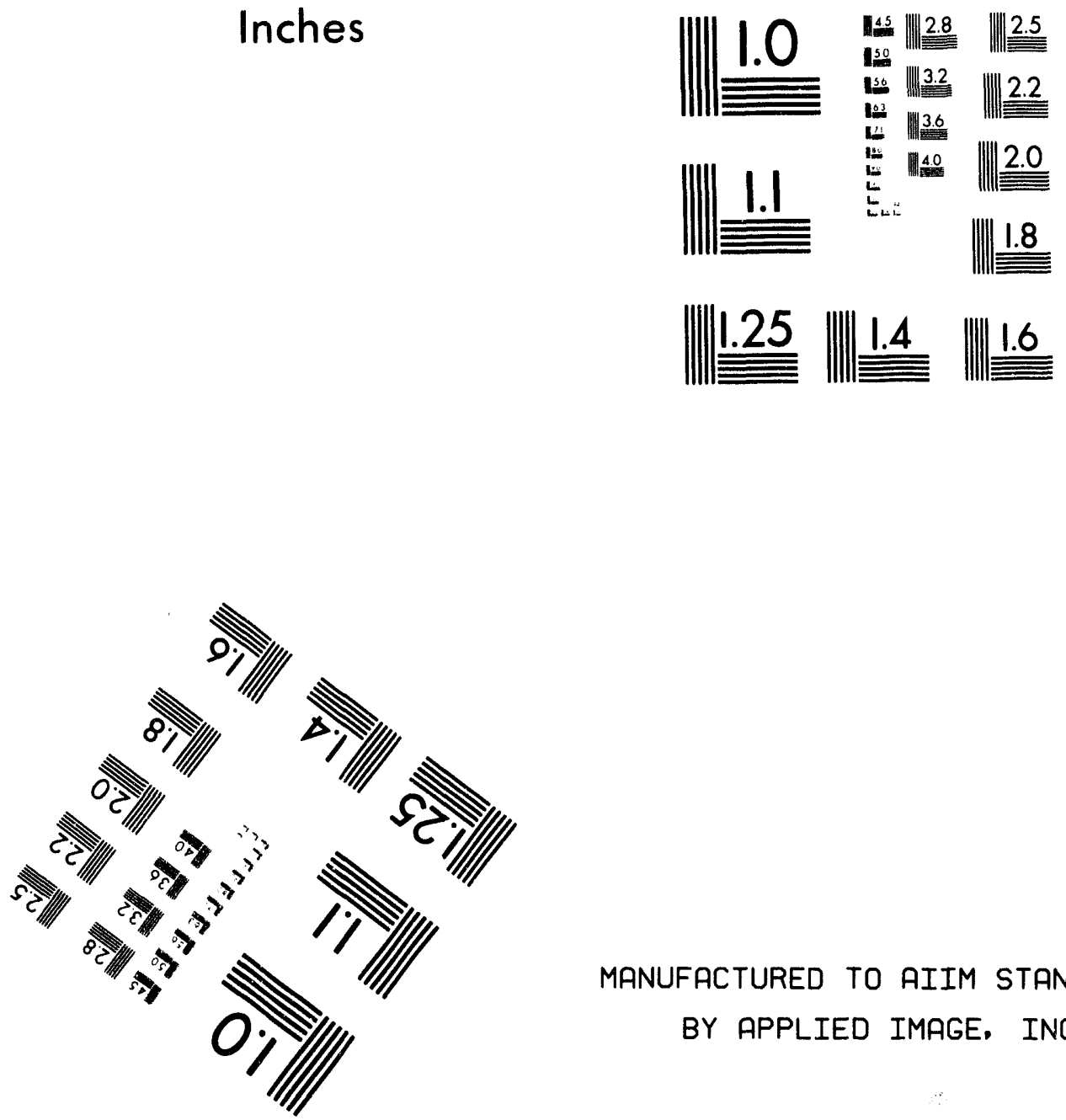

MANUFACTURED TO AIIM STANDARDS

BY APPLIED IMAGE, INC.

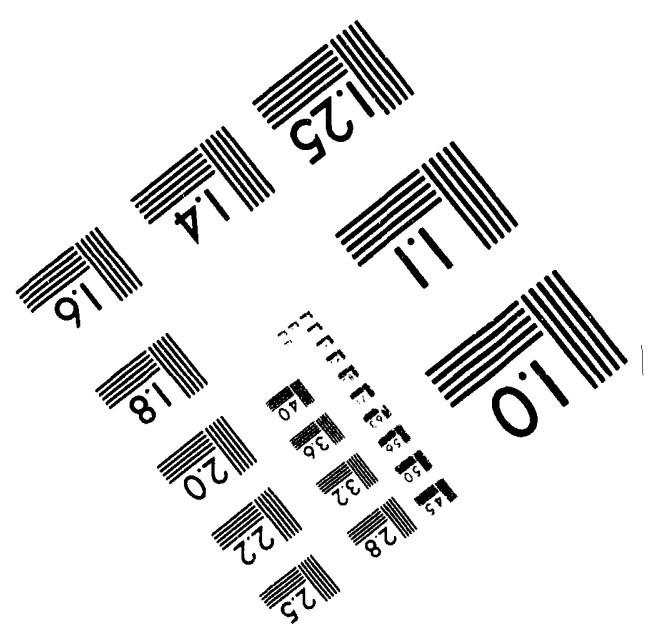



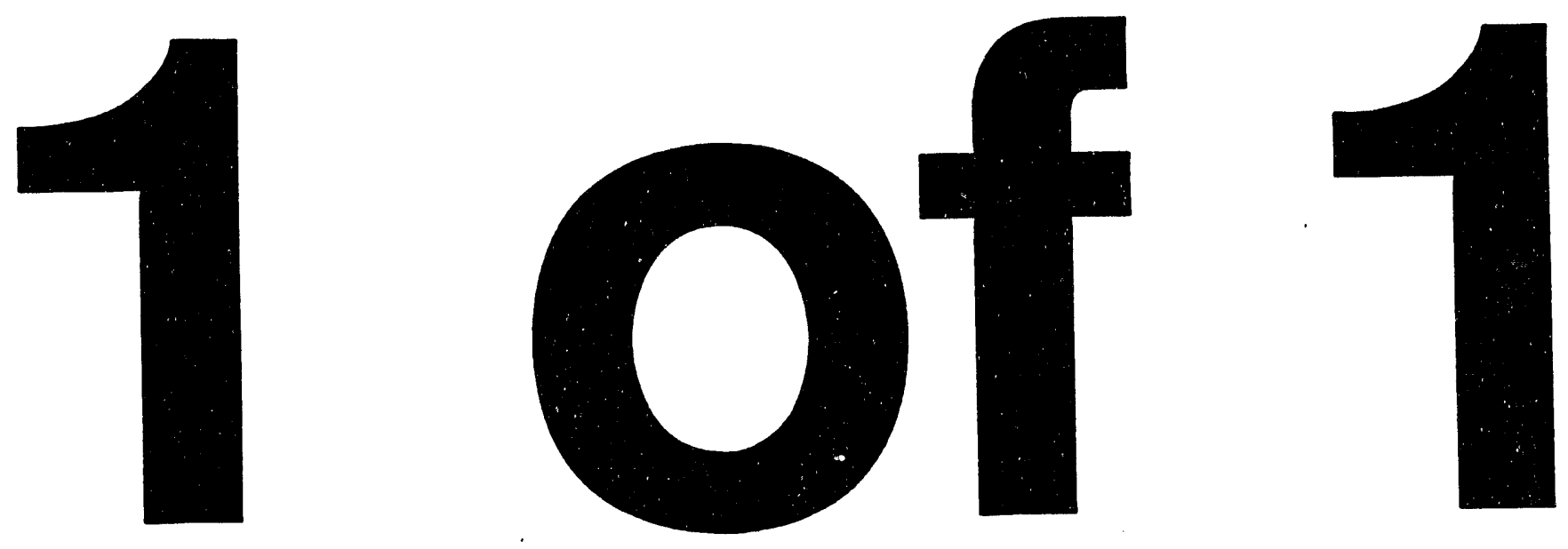


\title{
ANL/Mes/PP- -73014
}

\section{A Control Volume Method on an Icosahedral Grid for Numerical Integration of the Shallow-Water Equations on the Sphere *}

\author{
I-Liang Chern \\ Mathematics and Computer Science Division \\ Argonne National Laboratory \\ Argonne, IL 60439-4801
}

\begin{abstract}
Two versions of a control volume method on a symmetrized icosahedral grid are proposed for solving the shallow-water equations on a sphere. One version expresses of the equations in the 3-D Cartesian coordinate system, while the other expresses the equations in the northern/southern polar stereographic coordinate systems. The pole problem is avoided because of these expressions in both versions and the quasi-homogeneity of the icosahedral grid. Truncation errors and convergence tests of the numerical gradient and divergent operators associated with this method are studied. A convergence test for a steady zonal flow is demonstrated. Several simulations of Rossby-Haurwitz waves with various wave numbers are also performed.
\end{abstract}

AMS(MOS) Subject Classification. Primary 65M05. Secondary $76-08,76 \mathrm{C} 20$.

Key Words. control volume method, symmetrized icosahedral grid, bilinear interpolation, 3-D Cartesian coordinate system, polar stereugraphic coordinate system, shallow-water equations on a sphere, Rossby-Haurwitz waves, parallel computer.

This work was supported by the Applied Mathematical Sciences subprogram of the Office of Energy Research, U. S. Department of Energy, under Contract W-31-109-Eng-38.

\section{DISCLAIMER}

This report was prepared as an account of work sponsored by an agency of the United States Government. Neither the United States Government nor any agency thereof, nor any of their employees, makes any warranty, express or implied, or assumes any legal liability or responsibility for the accuracy, completeness, or usefulness of any information, apparatus, product, or process disclosed, or represents that its use would not infringe privately owned rights. Reference herein to any specific commercial product, process, or service by trade name, trademark, manufacturer, or otherwise does not necessarily constitute or imply its endorsement, recommendation, or favoring by the United States Government or any agency thereof. The views and opinions of authors expressed herein do not necessarily state or reflect those of the United States Government or any agency thereof.

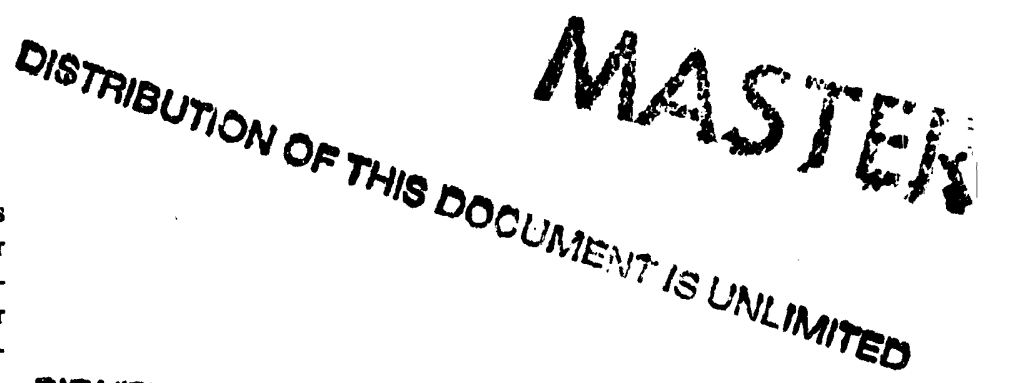

RIBUTION OF THIS DOCUMENT IS UNLIMITEA 


\section{Introduction}

General circulation models (GCMs) are numerical models to simulate largescale flow motions of the atmosphere. They usually consist of two parts: the dynamics and the physical parametrization. The dynamics part is addressed by a numerical algorithm that integrates the governing evolution equations of the atmosphere. The numerical grid scale associated with the algorithm is usually large (hundreds to thousands of kilometers). The subgrid physical processes (e.g., cloud, radiation) are then formulated as GCM parameters in terms of large-scale flow variables. This formulation, called physical parametrization, is more accurately formulaled ii the grid scale is small. (Certainly, the dynamics part certainly produces fewer errors.) Thus, for accuracy, it is always desirable to run simulations at as high a resolution as we can (based on the power of the state-of-the-art computers available). Current GCMs are rarely run at resolutions finer than $100 \mathrm{~km}$ for weather forecasting [21] and $400 \mathrm{~km}$ for climate simulations [29].

Parallel computers are now becoming available that offer peak speeds considerably faster than conventional supercomputers. For instance, the Intel Delta machine has 528 parallel processors each with a peak speed of 60 Mflops ( $10^{6}$ mathematical sperations per second). The number of parallel processors will go up to several thousands in a few years, and the speed of each processor is expected to become ten times faster within this decade. Thus, the computer speed can be expected to reach the teraflops ( $10^{12}$ mathematical operations per second) range soon. However, as the number of processors increases, the cost of coordinating the computation and data communication also increases. Thus, this hardware development brings a challenge to the design of numerical algorithms-the challenge of parallelism.

Most GCMs today use the spectral method. The spectral method is popular because of its spectral accuracy and its avoidance of the "pole problem." However, it also has the following well-known deficiencies. The $N^{3}$ computational complexity of the spectral transformation, where $N$ is the number of latitude lines, prohibits higher-resolution simulation. The solutions may show spurious oscillations, a deficiency that is particularly serious for the moisture field. Moreover, a recent study of the parallel scalability of the spectral transform method showed that the spectral method has a poor parallel efficiency, because of the global communication required in the spectral 
transformation $[9,30]$. Thus, alternatives that are suitable for massively parallel computers are needed. In a sequence of papers, we shall report on the search for such alternatives. In this search, the locality (local data communication) of an algorithm is one of our major concerns. Thus, we shall focus on explicit grid-point methods in which a fixed Eulerian grid is used. The finite difference and semi-Lagrangian methods are two such methods we shall consider. The grid-point methods are not only suitable for parallel computation, but potentially also suitable for coupling with ocean dynamics and for easier cooperation with the variation of topography.

The shallow-water equations are usually used as prototype equations for designing numerical algorithms for the general circulation of the atmosphere [3]. Mathematically, there are three well-known challenges in numerical integration of the shallow-water equations on a sphere, namely, the curvilinearity of the sphere, the spurious oscillations near large.gradients, and the multiple time scales of the solutions (the shallow-water equations support fast inertia-gravity waves and slow Rossby waves, the latter carrying most of the atmospheric energy); see [17, Chapter 3] or [4]. In this and a companion paper, we focus on the first challenge. The second and third challenges will be studied in future reports.

The first challenge raises three fundamental issues: what coordinate system should one use? what form of the equations should one choose (the primitive $u-v$ form or the vorticity-divergence form)? how shall one choose a grid system and perform numerical interpolation and differentiation on a sphere? We consider these issues below.

\subsection{Coordinate System}

A spherical coordinate system has traditionally been used in most numerical models for numerical integration of the shallow-water equations on a whole sphere (for example, for finite difference methods $[1,6]$, for finite element methods [7], for spectral methods [2], and for semi-Lagrangian methods [22]). The grid systems are usually built on the latitude-longitude plane (for example, the uniform grid in finite difference methods $[1,6,13]$ and the Gaussian grid in spectral methods [3] and semi-Lagrangian methods [18]). However, there are severe problems near the foles with the spherical coordinate system. Mathematically, at the poles, the Jacobian of the mapping from the latitude-longitude plane to a sphere is zero; the curvature terms 
waves) while the vorticity equation carries only slow waves (Rossby waves). Thus, the vorticity-divergence form can easily be fitted into the framework of semi-implicit methods to gain large time steps and, hence, to obtain efficiency. With respect to this second advantage, the time step obtained by the use of the semi-implicit method is approximately four times larger: the jet stream can 'go up to $100 \mathrm{~m} / \mathrm{s}$, whereas the fast wave speed is at most $400 \mathrm{~m} / \mathrm{s}$. However, additional overhead is introduced: two Poisson equations must be solved for the stream function and the velocity potential. This changes the nature of the problem from a local domain of dependence (hyperbolicity of the shallow-water equations) to a global domain of dependence (ellipticity of the Poisson equation). and necessitates global data communication, which compromises efficient parallel execution. Thus, it is still questionable whether the simulation of atmospheric motions can be achieved efficiently by using a semi-implicit method on large-scale parallel machines. For this reason, we choose the primitive form of the equations for performing numerical integration.

\subsection{Grid System}

For the choice of an Eulerian grid, we have ruled out the use of the uniform grid and the Gaussian grid for their deficiency of the pole problem. There are two other possibilities: composite grids and quasi-homogeneous grids. A composite-grid system consists of several overlapping grid systems. Each of them covers a certain portion of the sphere and is the image of a regular grid on a plane. On these regular grids on the planes, it is usually easy to design a numerical algorithm that is high-order and upwinding. However, the interpolation required in the overlapping region creates a mapping problem: data must be interpolated from coarse grid to fine grid. This problem is due to the fact that the Euclidean metric which the regular grid is based on is different from the spherical metric induced by the mapping. We shall report on this issue in a separate paper in the future. Here, we focus on the other possibility, quasi-homogeneous grids.

A quasi-homogeneous grid is a grid induced by a triangulation on the sphere, which has the property that each triangle is nearly equilateral. There is no homogeneous grid on the sphere with more than 12 nodes. A nice example of a quasi-homogeneous grid is the icosahedral grid, which can be constructed from a spherical icosahedron by partitioning its 20 equilateral 
lem because the curvature terms of the momentum equations are singular at the poles.

Since the grid in our approach is not homogeneous, a symmetrization procedure is introduced to make the grid more regular. This procedure, together with a bilinear interpolation, reduces the spatial truncation error of our control volume method dramatically. A series of tests has been performed to examine the convergence of our method. They are the convergence tests of numerical gradient and divergence operators, the test of a steady zonal flow, and several simulations of the Rossby-Haurwitz waves with various wave numbers and at various resolutions. In these tests, the method conserves exactly the total mass, and only small percentages of the total energy and absolutely enstrophy are lost in 10-day simulations.

\section{The Shallow-Water Equations on the Sphere}

\subsection{In Spherical Coordinate System}

The shallow-water equations on a sphere, in the spherical coordinate system, are

$$
\begin{gathered}
\frac{d \phi}{d t}+(a \cos \theta)^{-1} \phi\left[u_{\lambda}+(v \cos \theta)_{\theta}\right]=0, \\
\frac{d u}{d t}-a^{-1} u v \tan \theta+(a \cos \theta)^{-1} \phi_{\lambda}-f v=0, \\
\frac{d v}{d t}+a^{-1} u u \tan \theta+a^{-1} \phi_{\theta}+f u=0,
\end{gathered}
$$

where $\lambda$ is the longitude; $\theta$ is the latitude; $a$ is the radius of the earth; $\phi$ is the geopotential; $u$ and $v$ are the eastward and northward velocity components, respectively; $f=2 \Omega \sin \theta$ is the Coriolis parameter; $\Omega$ is the angular velocity of the earth; and

$$
\frac{d}{d t}=\frac{\partial}{\partial t}+(a \cos \theta)^{-1} u \frac{\partial}{\partial \lambda}+a^{-1} v \frac{\partial}{\partial \theta} .
$$

By rescaling the time by $t^{\prime}=t / a$ and $f^{\prime}=a f$ and dropping the prime, (2.1)(2.3) with $a=1$ is the shallow-water equations on the unit sphere. The terms $u v \tan \theta$ and $u u \tan \theta$ in the momentum equations are called the curvature terms. They are singular at the poles and cause numerical difficulties there. 
where $m=2(1+q \sin \theta)^{-1}$ is the map factor, $q=1$ for the northern hemisphere and $q=-1$ for the southern hemisphere. The equator corresponds to $X^{2}+Y^{2}=4$. The range of $(X, Y)$ in each hemisphere is $X^{2}+Y^{2} \leq 4$, in which the map factor $m$ is between 1 and 2. The shallow-water equations in these coordinate systems in the flux form are

$$
\begin{aligned}
\frac{\partial}{\partial t}\left(m^{-2} \mathrm{U}\right) & +\frac{\partial}{\partial X}\left(m^{-1} \mathbf{F}\right)+\frac{\partial}{\partial Y}\left(m^{-1} \mathbf{G}\right)=m^{-2} \mathbf{H}, X^{2}+Y^{2} \leq 4 \\
\mathbf{U} & =\left[\begin{array}{c}
\phi \\
\phi U \\
\phi V
\end{array}\right], \quad \mathbf{F}=\left[\begin{array}{c}
\phi U \\
\phi U^{2}+p \\
\phi U V
\end{array}\right], \quad \mathbf{G}=\left[\begin{array}{c}
\phi V \\
\phi U V \\
\phi V^{2}+p
\end{array}\right], \\
\mathbf{H} & =\mathbf{H}_{1}+\mathbf{H}_{2}+\mathbf{H}_{3} \\
& =q f \phi\left[\begin{array}{c}
0 \\
V \\
-U
\end{array}\right]-\frac{\phi(X V-Y U)}{2}\left[\begin{array}{c}
0 \\
V \\
-U
\end{array}\right]-\frac{p}{2}\left[\begin{array}{c}
0 \\
X \\
Y
\end{array}\right],
\end{aligned}
$$

where $p=\phi^{2} / 2$ and $U, V$ are the X-, Y-component of the wind, respectively. Notice that the curvature terms $\mathbf{H}_{2}, \mathbf{H}_{3}$ as well as the velocity components $U, V$ are regular now.

\section{Icosahedral Grids on a Sphere}

An icosahedral grid on a sphere can be constructed from a spherical icosahedron that has 12 nodes and 20 equilateral triangular surfaces. A straightforward way of constructing grid points in these spherical triangles is to partition a planar equilateral triangle uniformly and then project this partition radially onto the sphere. Unfortunately, such a construction gives a large variation of distance between grid points, which leads to increased errors in finite difference methods [27]. To minimize such a variation, Sadourny et al. [20] and Williamson [24] proposed two different but similar constructions based on geodesic arguments.

Our approach is a symmetrization of Sadourny et al.'s construction. According to Sadourny et al. [20] (see Figure 1), the great circle arcs $A B$ and $A C$ of the spherical triangle $\triangle A B C$ are divided into $N$ equal arcs to give $B_{1}, \cdots, B_{N}$ and $C_{1}, \cdots, C_{N}$. The great circle arcs $B_{i} C_{i}$ are then divided into 
sphere.

\section{A Control Volume Method}

In this section, we propose two versions of a control volume method on the above icosahedral grid to solve the shallow-water equations on the sphere. The 3-D version uses the 3-D Cartesian coordinate system, while the 2-D version uses the north/south polar stereographic coordinate systems. In Figure 2, we consider a spherical control area element $A$ (defined in the preceding section) centered at a grid point $P_{0}$. Let $L$ denote the number of triangles surrounding $P_{0}$, where $L=5$ or 6 ; let $C_{i}$ denote the geodesic curve connecting the centroids of the surrounding triangles.

\subsection{3-D Version}

We first integrate (2.7) without the centripetal force over $A$ and apply Stokes's theorem to obtain

$$
\iint_{A} \hat{\mathrm{U}}_{t} d A=\sum_{i=1}^{L} \int_{C_{i}} \mathbf{F} \cdot \vec{n} d s+\iint_{A} \mathbf{H}_{1} d A .
$$

Here, $\vec{n}$ is the inner normal of the geodesic curve $C_{i}$ on the sphere, and $\widehat{\mathrm{U}}=(\phi, \hat{\vec{V}})^{t}$ is an intermediate state. Then, the intermediate velocity $\hat{\vec{V}}$ is projected onto the tangent space of the sphere. This takes care of the contribution of the centripetal force.

Next, we discretize (4.1) using the leap-frog method in the temporal direction and using the midpoint rule for the line integrals in the spatial direction. Let us denote by $U^{n}\left(P_{0}\right)$ the average state of $U$ at time step $n$ in the control area element $A$ centered at $P_{0}$; by $M_{i}$ the midpoint of the geodesic curve $C_{i} ;$ and by $\Delta t, \Delta s_{i}$, and $\Delta A$, respectively, the temporal mesh size, the arc length of the geodesic curve $C_{i}$ and the surface area of $A$. Then the intermediate state $\hat{\mathrm{U}}^{n+1}=\left(\phi^{n+1}, \hat{\vec{V}}^{n+1}\right)^{t}$ is defined by

$$
\begin{aligned}
\hat{\mathrm{U}}^{n+1}\left(P_{0}\right)= & \mathrm{U}^{n-1}\left(P_{0}\right) \\
& +\left.\frac{2 \Delta t}{\Delta A} \sum_{i=1}^{L} \mathbf{F}\left(\overline{\mathrm{U}}^{n}\left(M_{i}\right)\right) \cdot \vec{n}\right|_{M_{i}} \Delta s_{i}
\end{aligned}
$$


For time-evolution problems, the second-order truncation error in the firstorder region may become negative definite. Thus the following artificial dissipation is added to $F\left(\bar{U}^{n}\left(M_{i}\right)\right)$ of (4.2) to stabilize the scheme:

$$
\left.\mu\left(U^{n-1}\left(P_{i}\right)-U^{n-1}\left(P_{0}\right)\right) \otimes \vec{n}\right|_{M_{i}}
$$

where $\otimes$ denotes the tensor product operation. The coefficient of the artificial viscosity $\mu$ is typically chosen to be 2 . The following temporal filter is also introduced for time evolution simulations, as it usually is for a leap-frog scheme [12]:

$$
\tilde{U}^{n}=\mathbf{U}^{n}+\alpha\left(\tilde{U}^{n-1}-2 U^{n}+U^{n+1}\right) .
$$

Here, $\tilde{U}$ is the filtered state. The coefficient $\alpha$ is chosen to be 0.01 , which is smaller than 0.06 used in the community climate model (CCM1) [28].

The artificial dissipation term is certainly not ideal, since the low-order region of the spatial discretization covers only very small area. It is desirable to have a dissipation term that is second order in the low-order region and that gradually becomes higher order in the central area of the principal triangles. We have investigated some schemes with weighted dissipation but found that they did not help much in reducing the dissipation of the total energy and total enstrophy.

\subsection{2-D Version}

We use the northern- (resp. southern-) polar stereographic coordinate system (2.10) for grid points with latitude $\theta \geq 0$ (resp. $\theta<0$ ). Since the area element $d A$ on the sphere is $q m^{-2} d X \wedge d Y$ in this coordinate system, we multiply (2.11) by $q$, then integrate it over a control area element $A$ to obtain

$$
\iint_{A} \mathrm{U}_{t} d A=\sum_{i=1}^{L} \int_{C_{i}}\left(\mathrm{~F}(\mathrm{U}) n_{X}+\mathrm{G}(\mathrm{U}) n_{Y}\right) d s+\iint_{A} \mathrm{H} d A
$$

Here, we have applied Green's theorem to the divergence term; $\left(n_{X}, n_{Y}\right)$ are the inner normal $\vec{n}$ represented in the polar stereographic coordinate system. They are given by

$$
n_{X}=-q m^{-1} \frac{d Y}{d s}
$$


where $\langle$,$\rangle denotes for the inner product. On the other hand, the 2-D$ formulation yields

$$
b\left(<\vec{V}\left(P_{0}\right), \vec{e}_{X}\left(P_{0}\right)>,<\vec{V}\left(P_{i}\right), \vec{e}_{X}\left(P_{i}\right)>,<\vec{V}\left(P_{i \pm 1}\right), \vec{e}_{X}\left(P_{i \pm 1}\right)>\right) .
$$

The difference between (4.14) and (4.15) is simply the bilinear interpolation of the scalar field $\left\langle\vec{V}(P), \vec{e}_{X}(P)-\vec{e}_{X}\left(M_{i}\right)\right\rangle$ at $M_{i}$. Thus it is $O\left(h^{2}\right)$. Similarly, we also have an $O\left(h^{2}\right)$ difference between (4.14) and (4.15) when the southern-polar stereographic coordinate system is used in (4.15). Thus, there is an $O\left(h^{2}\right)$ difference in the midstate $\bar{U}$ and, hence, in the fluxes at $M_{i}$, when they are computed in the two different polar stereographic coordinate systems. Therefore, the 2-D version is not conservative. However, we demonstrate in the test section that there is only very little difference between the 2-D results and the 3-D results. Thus, the difference of the flux calculations in the two polar stereographic coordinate systems has very little influence on our low-order control volume method.

\section{Convergence Tests}

In this section, we perform a series of tests to examine the convergence of our method. The first test studies error resulting from the spatial discretization; specifically, we conduct convergence tests of our numerical gradient and divergence operators, independent of the shallow-water equations. The second test is a convergence test of steady zonal flow, which mimics a jet stream in the northern hemisphere [3]. The third test comprises several 10-day simulations of Rossby-Haurwitz waves with various wave numbers. These simulations were proposed by Phillips [16] and have been used by many researchers. In this test, the conservation of mass, total energy, and enstrophy are also investigated.

\subsection{Convergence Tests of Numerical Gradient and Diver- gence Operators}

This test studies error from the spatial discretization, due to the inhomogeneity of the grid. The basic operations of our control volume method are a numerical gradient operator for scalar fields and a numerical divergence operator for vector fields. 
Table 3: Convergence test of the 3-D scheme with the nonsymmetric grid as used in Table 2 and the bilinear interpolation for $\nabla p$ with $p=x_{3}$

\begin{tabular}{||c||c|c|c||}
\hline $\mathrm{N}$ & $L^{1}$ & $L^{2}$ & $L^{\infty}$ \\
\hline \hline 8 & $9.8 \mathrm{e}-03$ & $2.9 \mathrm{e}-03$ & $1.6 \mathrm{e}-02$ \\
\hline 16 & $2.7 \mathrm{e}-03$ & $8.0 \mathrm{e}-04$ & $6.6 \mathrm{e}-03$ \\
\hline 32 & $7.2 \mathrm{e}-04$ & $2.2 \mathrm{e}-04$ & $4.3 \mathrm{e}-03$ \\
\hline 64 & $1.9 \mathrm{e}-04$ & $6.5 \mathrm{e}-05$ & $2.4 \mathrm{e}-03$ \\
\hline
\end{tabular}

Table 4: Convergence test of the numerical gradient operator with Sadourny et al.'s grid and the linear interpolation. $p=x_{3}$

\begin{tabular}{||c||r|r|r||}
\hline $\mathrm{N}$ & $L^{1}$ & $L^{2}$ & $L^{\infty}$ \\
\hline \hline 8 & $7.8 \mathrm{e}-03$ & $2.6 \mathrm{e}-03$ & $1.8 \mathrm{e}-02$ \\
\hline 16 & $2.1 \mathrm{e}-03$ & $8.2 \mathrm{e}-04$ & $1.1 \mathrm{e}-02$ \\
\hline 32 & $5.6 \mathrm{e}-04$ & $2.7 \mathrm{e}-04$ & $5.7 \mathrm{e}-03$ \\
\hline 64 & $1.4 \mathrm{e}-04$ & $9.0 \mathrm{e}-05$ & $2.9 \mathrm{e}-03$ \\
\hline
\end{tabular}

other hand, Table 3 shows that, with the same grid but with a bilinear interpolation being used instead, the numerical gradient operator does converge in the maximum norm. These two tables demonstrate the sensitivity of ihe linear interpolation to the symmetry of the grid: the distortion of the grid can cause an $O(h)$ error in the linear interpolation.

Now we compare the error of the numerical gradient operator from other finite difference schemes on an icosahedral grid and our scheme. In the previous work, only the linear interpolation was used $[20,25,26,14]$. Table 4 uses Sadourny et al.'s grid and the linear interpolation [20, 14]. Table 5 uses our symmetric grid and the bilinear interpolation. We observe that, by symmetrizing the grid and using the bilinear interpolation, we gain almost one more digit of precision in the maximum norm.

The convergence rate is only first order in the maximum norm. However, the second-order convergence rate in $L^{1}$ reveals that this first order region covers only $O(h)$ area. Figure 3 shows that this first-order region lies only on the edges of the 20 principal triangles. The peak of the error is found at 
Table 6: Convergence test of the numerical divergence operator with the symmetrized grid-3-D version, $\vec{V}=\left(1-x_{1}^{2},-x_{1} x_{2},-x_{1} x_{3}\right)$

\begin{tabular}{||c||c|c|c||}
\hline $\mathrm{N}$ & $L^{1}$ & $L^{2}$ & $L^{\infty}$ \\
\hline \hline 8 & $5.4 \mathrm{e}-03$ & $1.9 \mathrm{e}-03$ & $2.0 \mathrm{e}-02$ \\
\hline 16 & $1.5 \mathrm{e}-03$ & $5.8 \mathrm{e}-04$ & $1.1 \mathrm{e}-02$ \\
\hline 32 & $3.9 \mathrm{e}-04$ & $1.9 \mathrm{e}-04$ & $5.8 \mathrm{e}-03$ \\
\hline 64 & $1.0 \mathrm{e}-04$ & $6.4 \mathrm{e}-05$ & $3.0 \mathrm{e}-03$ \\
\hline
\end{tabular}

and the numerical divergence of a vector field $\vec{V}$ is given by

$$
-\left.\frac{1}{\Delta A} \sum_{1} m\left(U n_{X}+V n_{Y}\right)\right|_{M_{i}} \Delta s_{i}
$$

where $U$ and $V$ are respectively the $X-$ and $Y$-components of $\vec{V}$ in the polar stereographic coordinate system. Tables 7-9 tabulate the results of the convergence tests of the numerical gradient operator and the numerical divergence operator on our symmetrized icosahedral grid. These results are similar to the $3-D$ results except that the $2-D$ version seems to converge slightly faster.

Table 7: Convergence test of the numerical gradient operator-2-D version. $p=x_{1}$.

\begin{tabular}{||l||r|r|r||}
\hline $\mathrm{N}$ & $L^{1}$ & $L^{2}$ & $L^{\infty}$ \\
\hline \hline 8 & $6.2 \mathrm{e}-03$ & $1.8 \mathrm{e}-03$ & $9.1 \mathrm{e}-03$ \\
\hline 16 & $1.6 \mathrm{e}-03$ & $4.6 \mathrm{e}-04$ & $2.5 \mathrm{e}-03$ \\
\hline 32 & $3.9 \mathrm{e}-04$ & $1.2 \mathrm{e}-04$ & $8.6 \mathrm{e}-04$ \\
\hline 64 & $9.9 \mathrm{e}-05$ & $3.0 \mathrm{e}-05$ & $4.3 \mathrm{e}-04$ \\
\hline
\end{tabular}




\subsection{Convergence Test for a Steady Zonal Flow}

A trivial solution of the shallow-water equations is the following steady zonal flow:

$$
\begin{gathered}
u=u(\theta), v=0, \\
\phi_{\theta}=-u^{2} \tan \theta-2 a \Omega \sin \theta u .
\end{gathered}
$$

This solution is usually used as the first test candidate for the validity of a numerical algorithm. We present the test given in [3], where the zonal flo:v is defined ae follows:

$$
\begin{gathered}
u(\theta)=u_{0} b(x) b\left(x_{e}-x\right) e^{4 / x_{e}}, v=0, \\
b(x)= \begin{cases}0, & x \leq 0 \\
e^{-1 / x}, & x>0\end{cases} \\
\phi=\phi_{0}-\int_{-\pi / 2}^{\theta}\left(2 a \Omega \sin \theta^{\prime}+u \tan \theta^{\prime}\right) u d \theta^{\prime} .
\end{gathered}
$$

The parameter values are chosen to be $u_{0}=40 \mathrm{~m} / \mathrm{s}, \phi_{0}=2.94 \times 10^{4} \mathrm{~m}^{2} / \mathrm{s}^{2}$, $a=6.371<2 \times 10^{6} \mathrm{~m}$, and $\Omega=7.292 \times 10^{-5} / \mathrm{s}$. This solution mimics a zonal jet stream in the northern hemisphere. Because of the steep gradient of this jet boundary, this solution is a good candidate to detect possible numerical instability. In the computation, the numerical viscosity and the time-filtering coefficient are chosen to be $\mu=1$ and $\alpha=0.005$ respectively; the Courant number is 0.6 . Tables 10 and 11 shows the relative errors of the $\phi$ and the wind fields in various norms for various resolutions after a 5-day simulation. These tables clearly show that the method is only first order. The cause is the low-order artificial dissipation term (4.7). We also see that there is not much difference between the 2-D result and the 3-D result. Figure 4 shows the geopotential of this zonal flow at day 0 and day 5

Table 10: Convergence test of a zonal flow-3-D version

\begin{tabular}{||c||c|c|c||c|c|c||}
\hline $\mathrm{N}$ & $\Delta \phi, L^{1}$ & $\Delta \phi, L^{2}$ & $\Delta \phi, L^{\infty}$ & $\Delta \vec{V}, L^{1}$ & $\Delta \vec{V}, L^{2}$ & $\Delta \vec{V}, L^{\infty}$ \\
\hline \hline 8 & $5.62 \mathrm{e}-03$ & $2.13 \mathrm{e}-03$ & $2.47 \mathrm{e}-02$ & $4.91 \mathrm{e}-02$ & $1.97 \mathrm{e}-02$ & $2.01 \mathrm{e}-01$ \\
\hline 16 & $3.31 \mathrm{e}-03$ & $1.28 \mathrm{e}-03$ & $1.39 \mathrm{e}-02$ & $2,59 \mathrm{e}-02$ & $1.10 \mathrm{e}-02$ & $1.14 \mathrm{e}-01$ \\
\hline 32 & $1.73 \mathrm{e}-03$ & $6.85 \mathrm{e}-04$ & $7.22 \mathrm{e}-03$ & $1.35 \mathrm{e}-02$ & $5.90 \mathrm{e}-03$ & $6.11 \mathrm{e}-02$ \\
\hline
\end{tabular}


therefore they almost satisfy the nondivergent barotropic equations. Thus, the Rossby-Haurwitz waves have been used by many researchers to test their algorithms for the shallow-water equations.

We perform 10-day simulations for wave number $R=4$ ai the two resolutions $N=16$ and $N=32$, respectively, and 10-day simulations for wave numbers 7 and 9 with $N=32$. The constants are chosen to be the same as Phillips's [16, pp. 339]. With these constants, the typical wind speed is from 65 to $99 \mathrm{~m} / \mathrm{s}$. The artificial viscosity and the filtering coefficient are chosen to be $\mu=2$ and $\alpha=0.01$. The Courant number is set to be 0.6 , which yields $\Delta r=10.6$ mins. for $N=16$, and 5.3 mins. for $N=32$ for these data. Below, we shall refer to the simulation with $N=16$ (resp. $N=32$ ) by N16 (resp. N32).

Figures 5 (a)-(e) show the geopotential of the N16 and N32 with wave number 4 using the 3-D scheme. Figures 6 (a)-(e) are the same plots using the 2-D scheme. These figures show that our method is more stable than previous schemes using a similar icosahedral grid ([26, Figs. 3 and 4$]$, and [25, Figs. 1 and 2]. It is difficult to compare our result with those of Masuda and Ohnishi's [14, Fig. 6], however, because different parameters are used. The Rossby-Haurwitz wave can be thought as a pertubation of a rigid-body rotation: in (5.17), the term $-a^{2} \omega \sin \theta$ is the rigid-body rotation, and the rest is the perturbation. It is an $O(1)$ perturbation in our test, whereas it is an $O\left(10^{-4}\right)$ perturbation in their test.

Regarding the accuracy problem, we find a nice agreement between the two resolutions at day 5 in both versions. At day 10 , however, a slight difference can be seen between N16 and N32: N32 has more structure (Figures $5(\mathrm{e})$ and $6(\mathrm{e})$ ), and the waves in N32 move slightly faster than those in N16 (Figs. 5 (c) and (e)). The wave speed $\nu$ given by (5.18) is $12.2^{\circ}$ per day. We measure the wave speed in our simulation by eye using the location difference of the waves at day 0 and 10 . The speed so measured for N32 is about $10.2^{\circ}$ per day with the 3-D scheme and $10.5^{\circ}$ per day with the $2-\mathrm{D}$ scheme. These results are consistent with the well-known slowup phenomena of the Rossby-Haurwitz waves [?]. The location difference of the waves between the two resolutions is about $6^{\circ}$ after 10 days. Compared with [26, Figs. 3 and 4], and [25, Figs. 1 and 2], which also performed simulations with different resolutions, our result is still better.

We claim that the wave-speed error in different resolutions is due to a 
where $\left.\vec{t}\right|_{M_{i}}$ is the tangent of $C_{i}$ at $M_{i}$ (see Figure 2). The initial vorticity is a street of vortex quadpoles, which are symmetric about the equator, with stronger strength at points $(A)$ and $(B)$, slightly weaker strength at points $(C)$ and $(D),+$ sign at $(A)$ and $(D)$, and - sign at $(C)$ and $(D)$. If no Coriolis force is present, then vortex (A) wuuld be moved up by $(C)$ as shown in Figures $8(a)-(f)$. The presence of the Coriolis force reverses such a motion: (C) is moved up by (A) (Fig. 7 (c)). The pairs of (A) and (B) form a street of vortex dipoles moving eastwa rd in the equatorial area, where the Coriolis effect is very small. This is similar to the motion of vortex dipoles in an ordinary fluid flow $[15,31]$. The presence of vortex (C) slows down the above motion of the dipolar-vortex street, because (A) and (C) also form a vortex dipole moving westward. This is the main mechanism of the motion of a Rossby-Haurwitz wave. For the cases of high wave number, we observe that this dipolar vortex street is unstable. It bifurcates to a Von Kámán vortex street. Also, vortex merging is observed in the later part of the simulation. In day 10 of the wave number 9 simulation, only 5 pairs of large vortices survive (Figure 9 ). The symmetry about the equator is broken because high-wave-number Rossby-Haurwitz waves are unstable. This bifurcation and vortex merging are expected to be sensitive to the vortex strength, the wave number, and the numerical viscosity. We also performed wave numbers 7 and 9 simulations by using the 2-D version of the method (Figures 10 and 11); very little difference was noted.

\section{Acknowledgments}

This research was supported by the DOE CHAMMP initiative. I am grateful to members of the CHAMMP Inter-Organizational Team, a collaboration involving Argonne National Laboratory, the National Center for Atmospheric Research, and Oak Ridge National Laboratory, for providing useful discussicns. I also thank Dr. Phillip Rasch for providing useful references and Dr. In-Young Lee for reading this manuscript and helpful discussions.

\section{References}

[1] A. Arakawa and V. R. Lamb, "Computational design of the basic dynamical progresses of the UCLA general circulation model," in Methods 
[12] H-O Kreiss and J. Oliger, "Comparison of accurate methods for the integration of hyperbolic equations," Tellus, 24, no. 3 (.1972), 199-215.

[13] Y. Masuda and H. Ohnishi, "An integration scheme of the primitive equation model with an icosahedral-hexagonal grid system and its application to the shallow-water equations," in Short- and Medium-Range Numerical Weather Prediction, WHO/IUGG NWP, Tokyo (1986) 317326.

[14] E. A. Overman and N. J. Zabusky, J. Fluid Mech. 125, 187 (1982).

[15] N. Phillips, "Numerical integration of the primitive equations on the hemisphere," Mon. Wea. Rev., 87, no. 9 (1959), 333-345.

[16] J. Pedlosky, Geophysical Fluid Dynamics, 2nd edition. Springer-Verlag, New York (1987).

[17] H. Ritchie, "Semi-Lagrangian advection on a Gaussian grid," Mon. Wea. Rev., 115 (1987) 608-619.

[18] C.-G. Rossby et al., "Relation between variations in the intensity of the zonal circulation of the atmosphere and the displacements of the semi-perminent centers of action," J. Marine Research, 2, (1939), 38-55.

[19] R. Sadourny, A. Arakawa, and Y. Minitz, "Integration of the nondivergent barotropic vorticity equation with an icosahedral-hexagonal grid for the sphere," Mon. Wea. Rev., 96, no. 6 (1968), 351-445.

[20] A. Simmons, "Some computational aspects of numerical weather prediction," Euro-physics Conference Abstracts, 14F (1990), 21.

[21] A. Staniforth and J. Côté, "Semi-Lagrangian integration schemes for atmospheric models - a review," Preprint, Recherche en prévision numérique, Service de l'environment atm phérique (1990).

[22] E. H. Vestine, W. L. Sibley, J. W. Kern, and J. L. Carlstedt, "Integral and spherical-harmonic analyses of the geomagnetic field for 1955.0, Part 2," J. Geomagnetism and Geoelectricity, 15, no 2 (Aug. 1963), 73-89.

[23] D. L. Williamson, "Integration of the barotropic vorticity equation on a spherical geodesic grid," Tellus, 10 (1968),642-653. 


\section{Figure Captions}

- Figure 1. Sadourny et al.'s construction of the geodesic grid points in a principal equilateral triangle [20]

- Figure 2. The geodesic construction of a control area element

- Figure 3. The absolute value of the error of $\nabla 1$ for $N=32$. Major errors are located on the edges of the 20 principal triangles.

- Figure 4. Simulation of a steady 2 nal jet with $N=8$ : (a) is the initial geopotential, (b) and (c) are the geopotential at day 5 using 3-D and 2-D methods respectively

- Figure 5. 3-D version. Geopotentials of 10-day simulations of a Rossby-Haurwitz wave at two different resolution, $N 16$ and $N 32$ : (a) is the initial data, (b) and (c) are the result of N16 at day 5 and 10, (d) and (e) are the results of N32 at day 5 and 10, and (f) is the geopotential of N32 at day 10 with doubled $\mu$ and $\alpha$.

- Figure 6. 2-D version. Same plots as Figure 5

- Figure 7. 3-D version. Vorticity of a 10-day simulation of a RossbyHaurwitz wave with $R=7$

- Figure 8. 3-D version. Vorticity of a 10-day simulation of a RossbyHaurwitz wave with $R=7$. Here, the Coriolis force is set to be 0 .

- Figure 9. 3-D version. Vorticity of a 10-day simulation of a RossbyHaurwitz wave with $R=9$.

- Figure 10. 2-D version. Geopotential and vorticity of a 10-day simulation of a Rossby-Haurwitz wave with $R=7$. Here, the Coriolis force is set to be 0 .

- Figure 11. 2-D version. Geopotential and vorticity of a 10-day simulation of a Rossby-Haurwitz wave with $R=9$. 

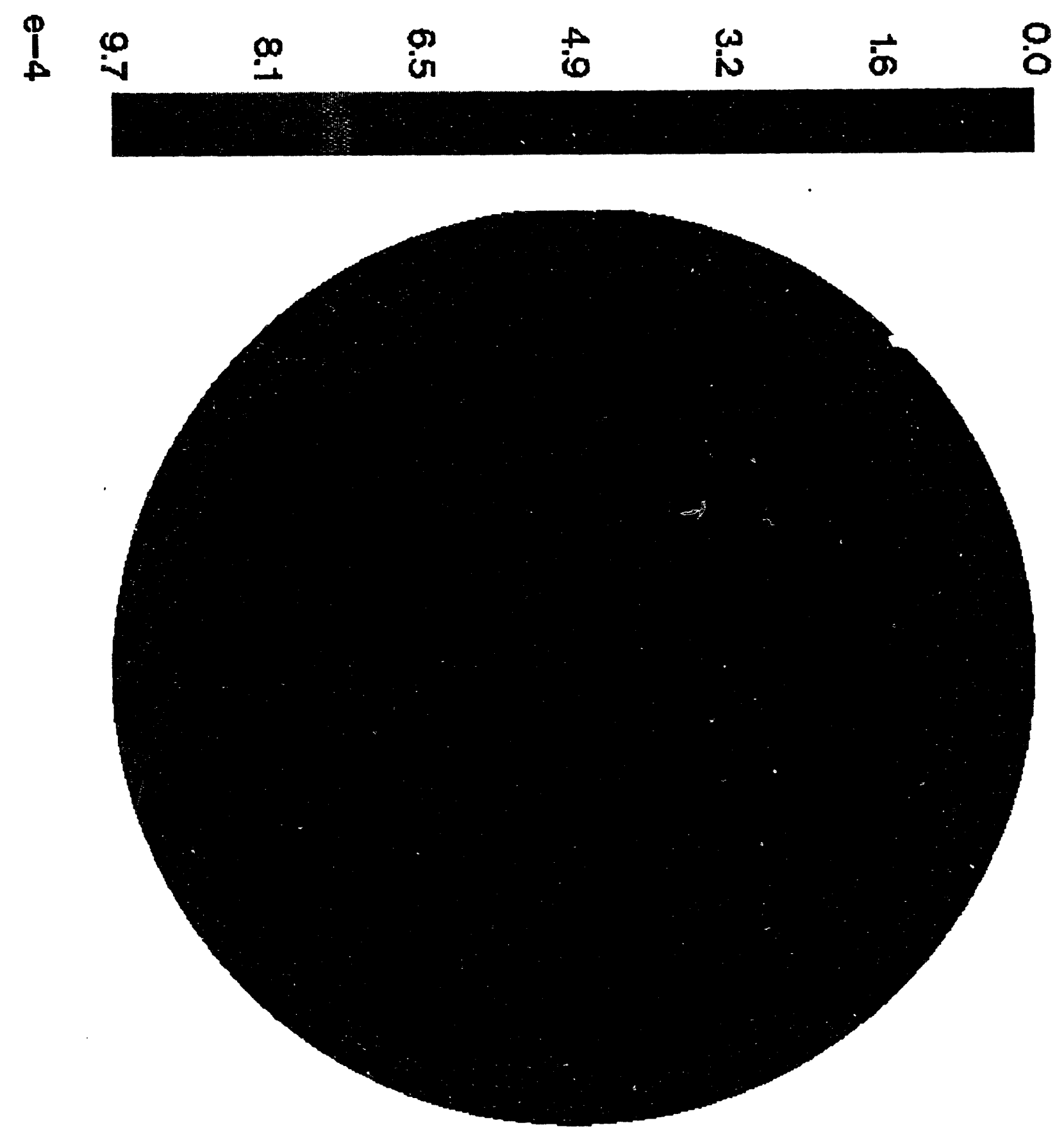

$\tau \varepsilon=N$ 'L pej6 jo 


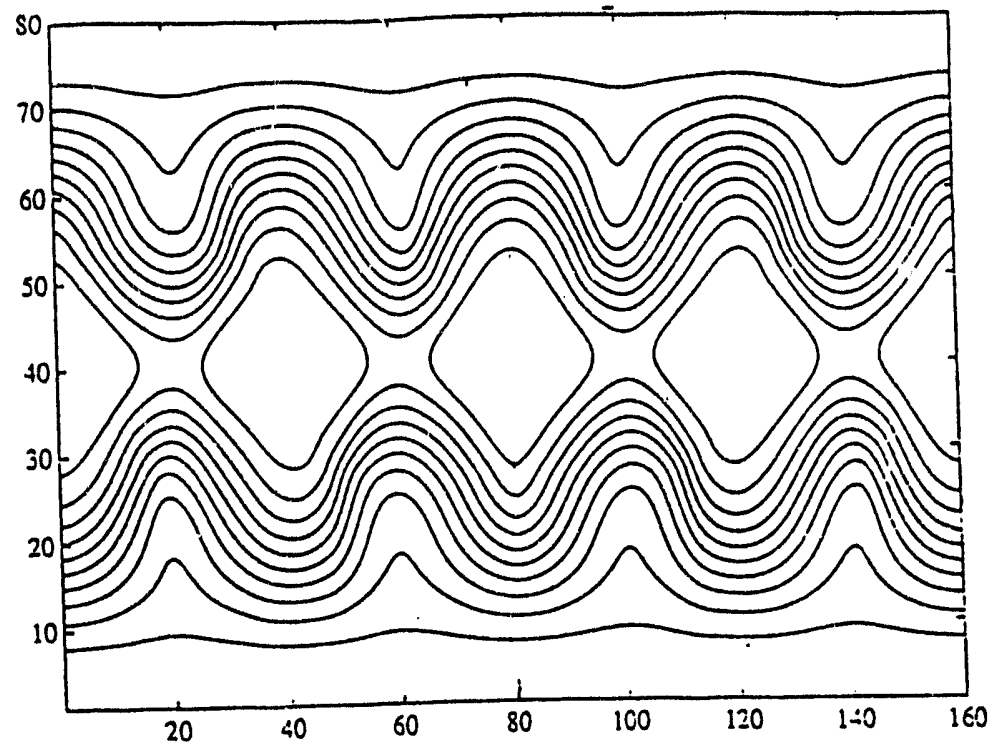

3-T Version

(a) day 0

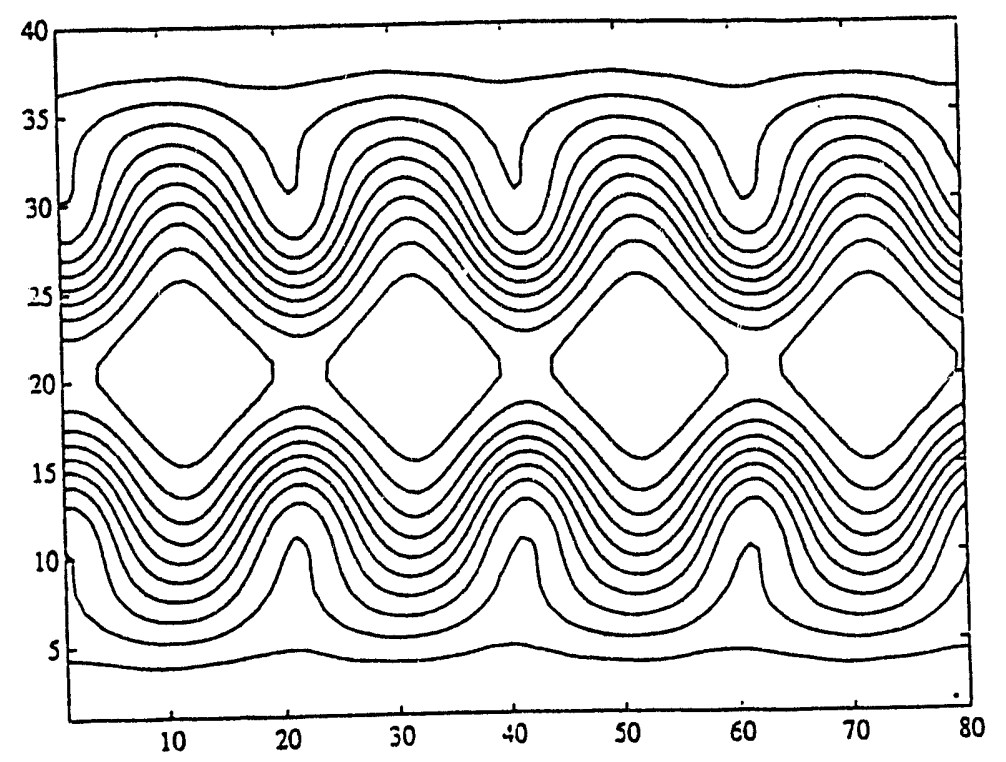

(b) N16, day 5

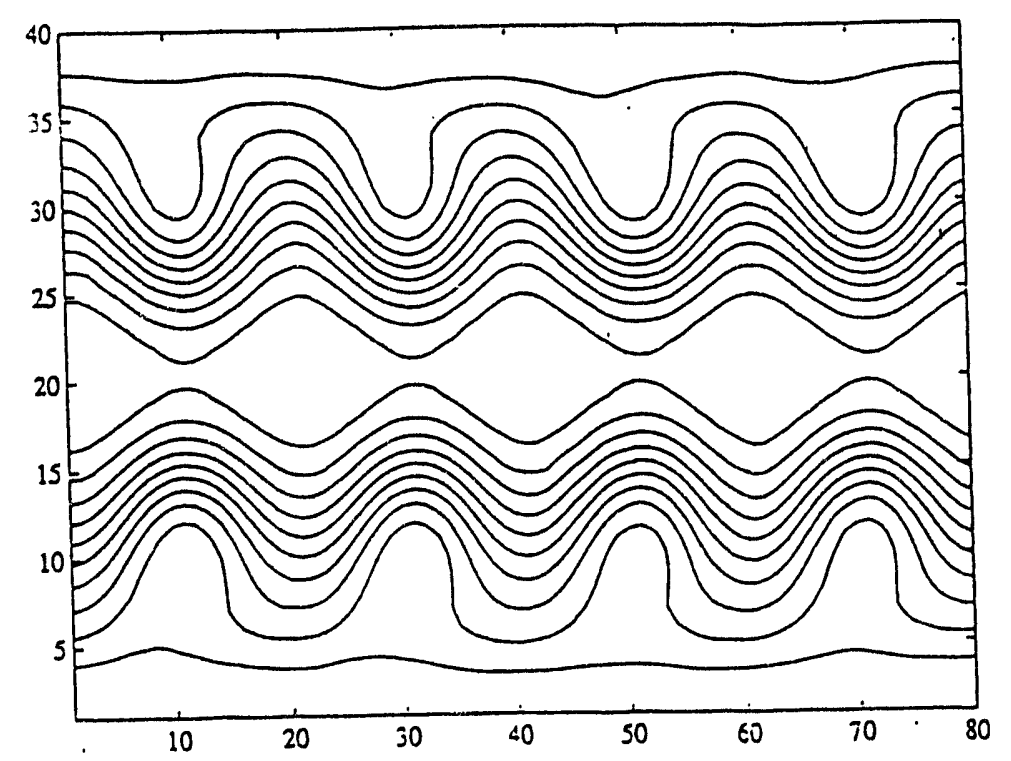

(c) $\mathrm{N} 16$, day 10

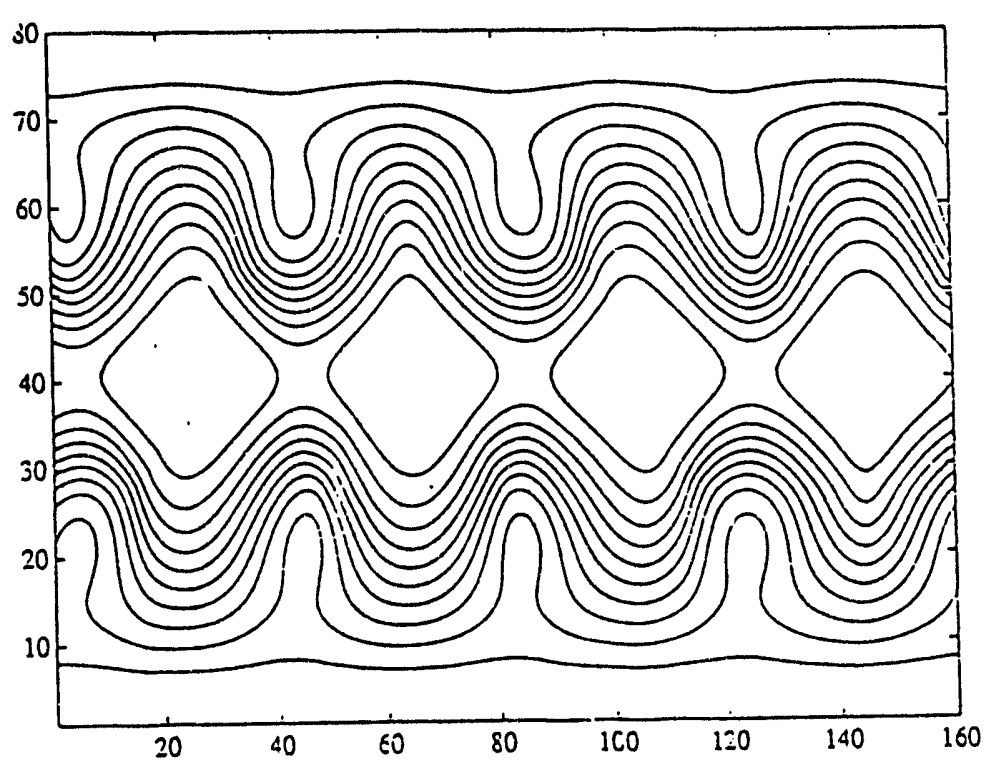

(d) N32, day 5

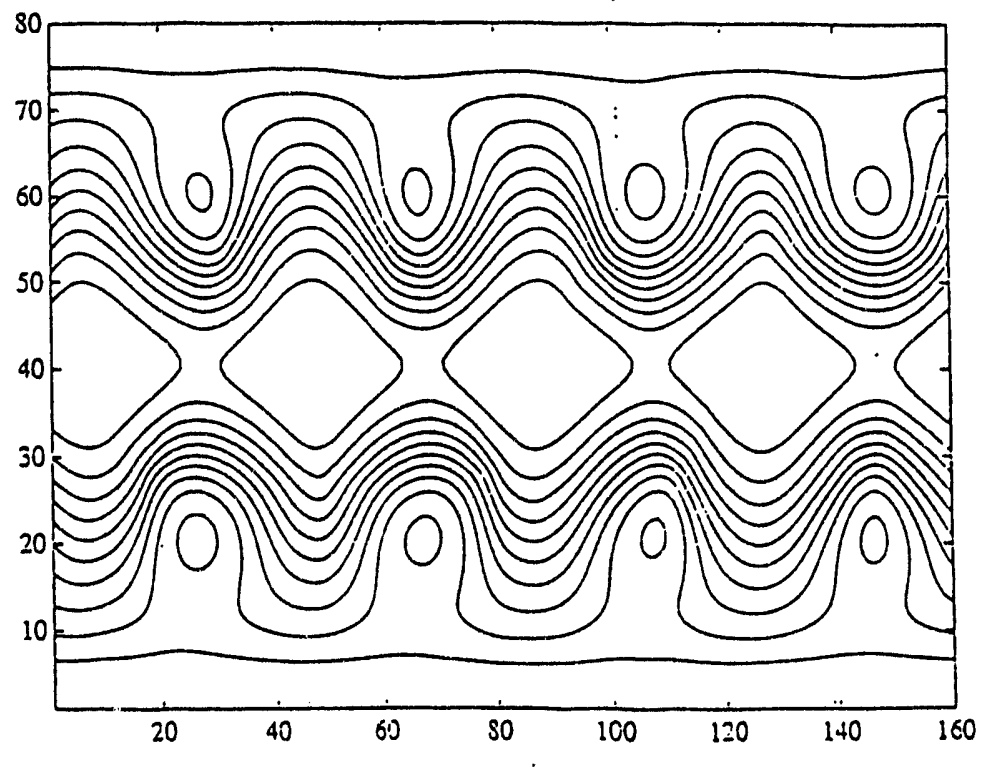

(e) N32, day 10 


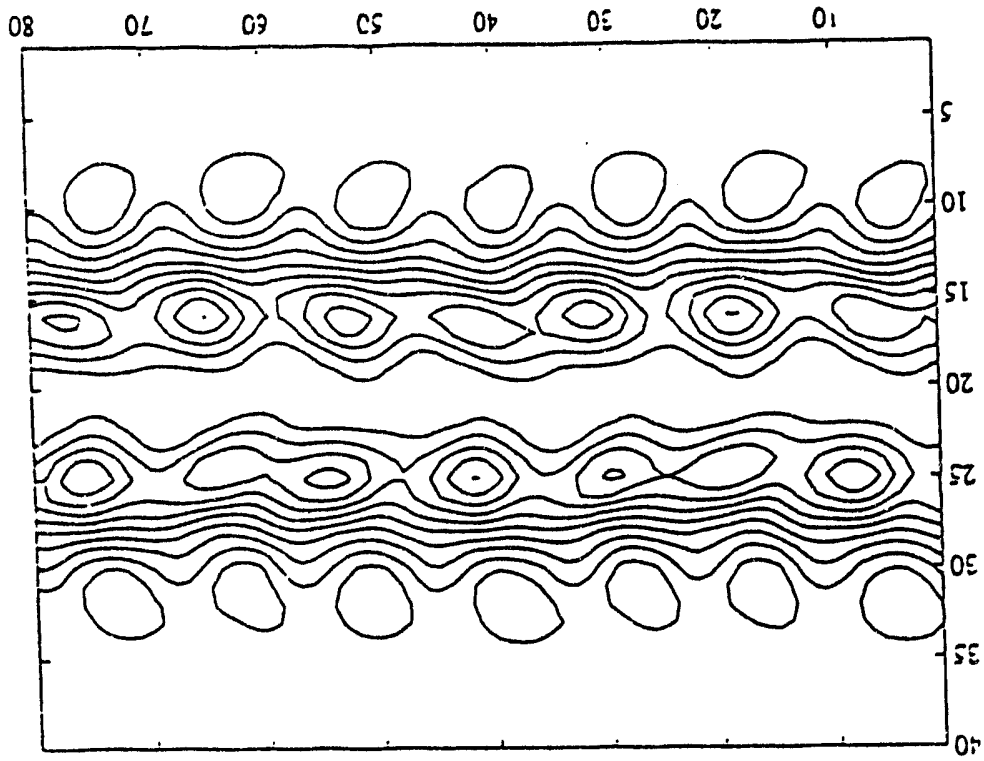

$8 \operatorname{\kappa ep~(\partial )~}$

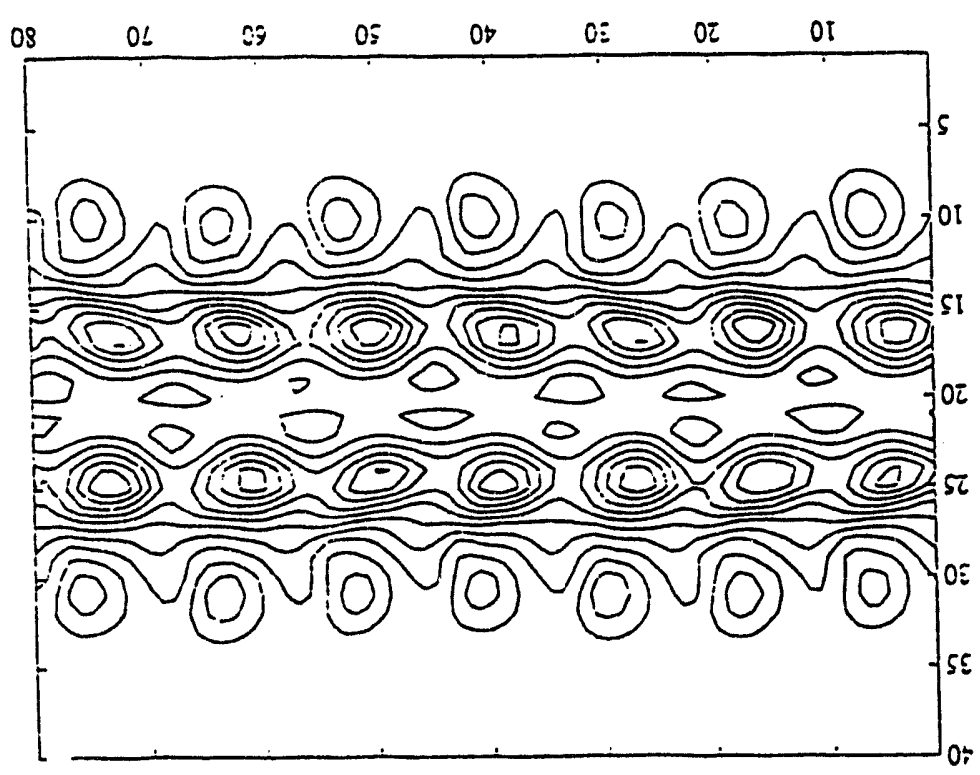

$9 \operatorname{\kappa ep}(p)$

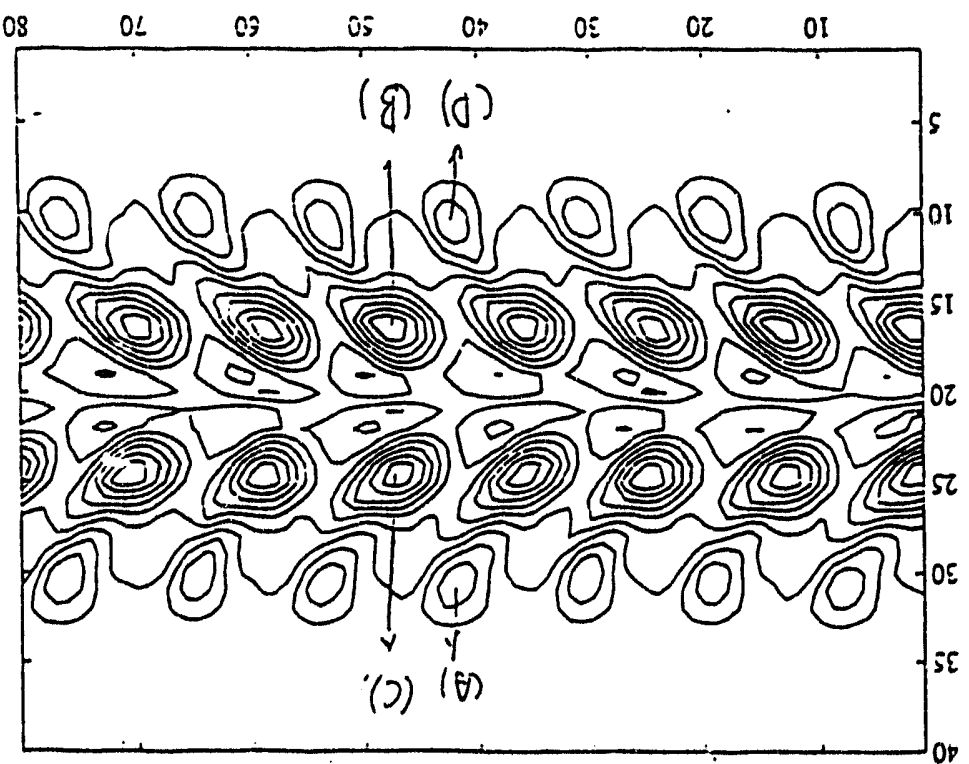

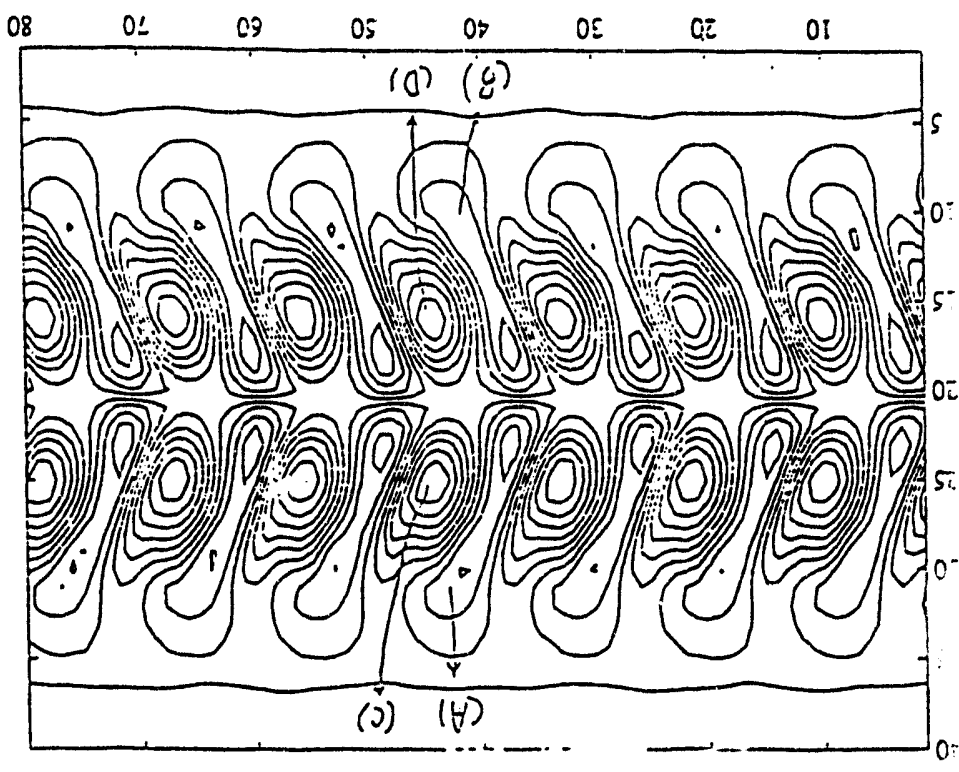

$\tau \operatorname{Sep}(q)$

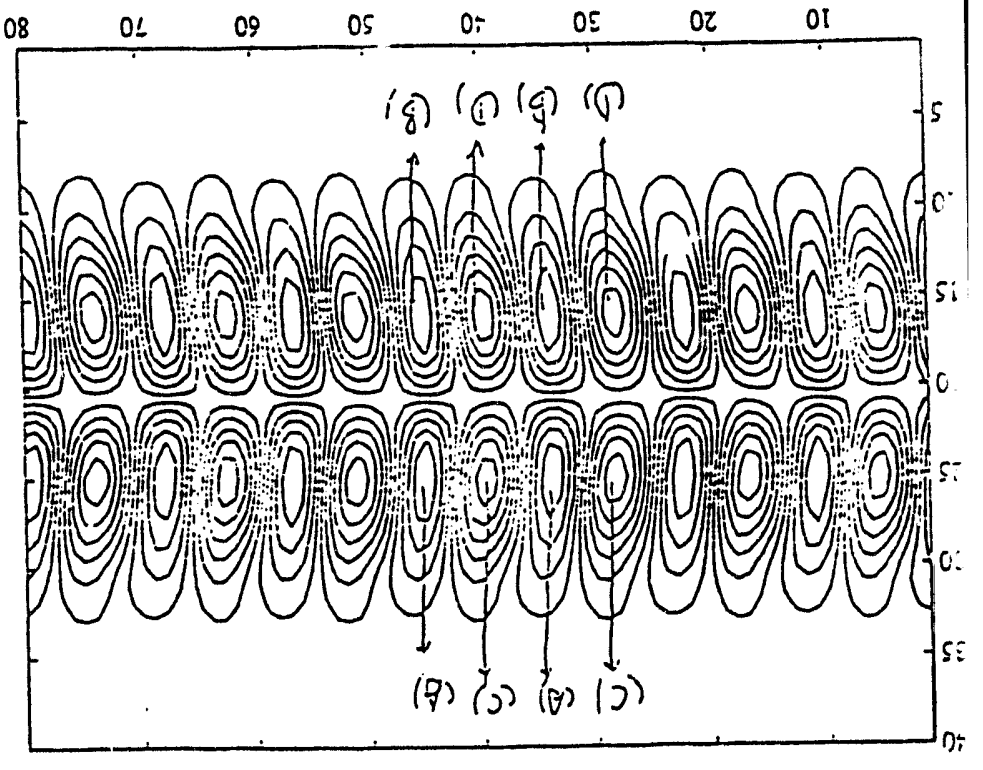

$$
0 \operatorname{sep}(\mathrm{e})
$$

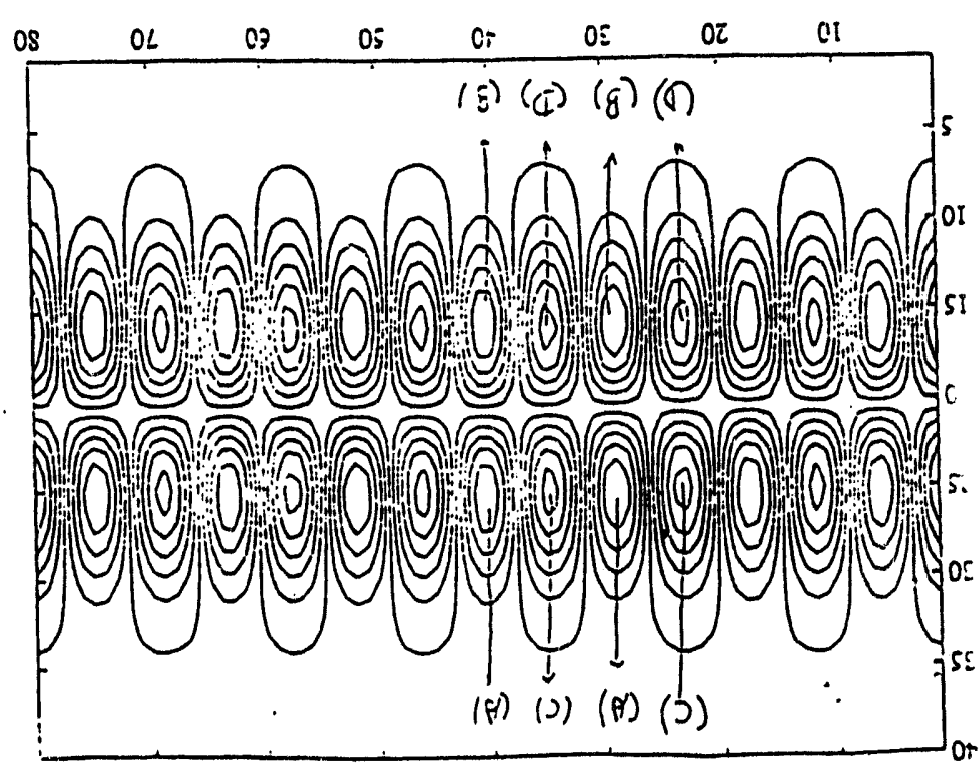



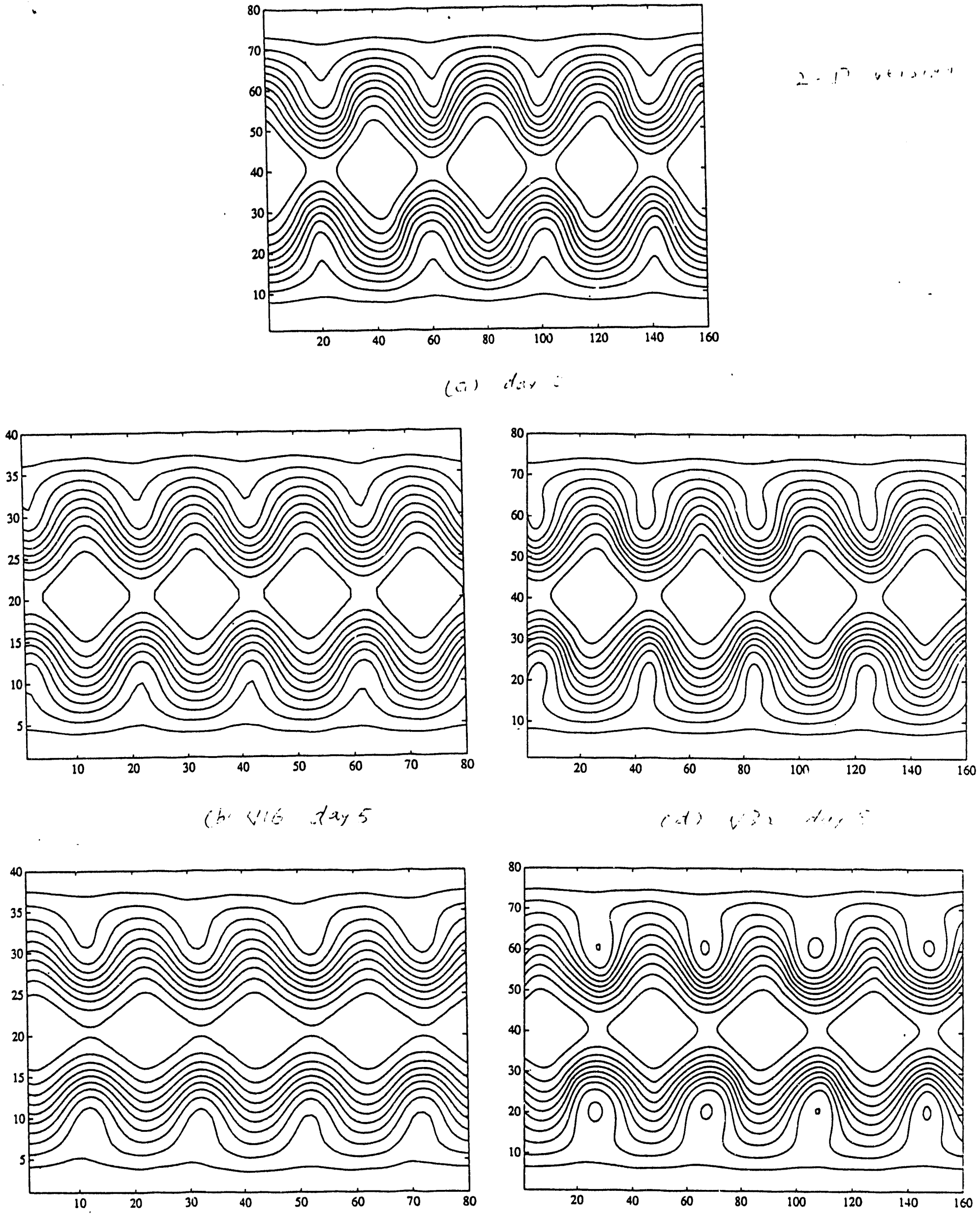

vile, iay. Fragure

$\therefore, 6 \because 1, \therefore=$ 


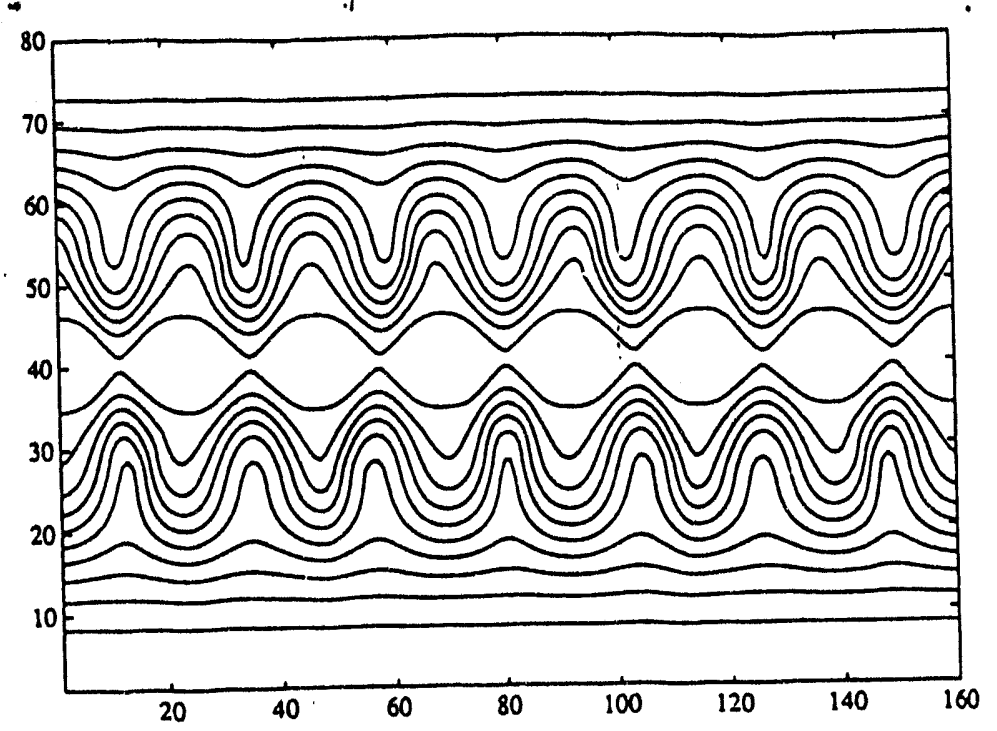

(a) day

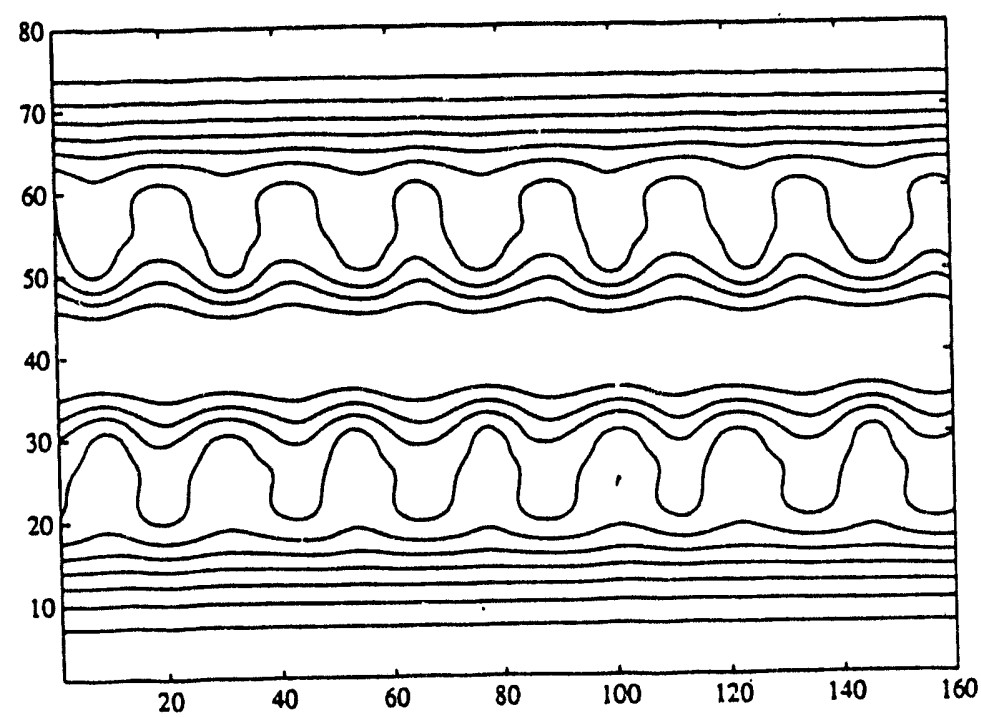

ibi $-4 a \cdots s$

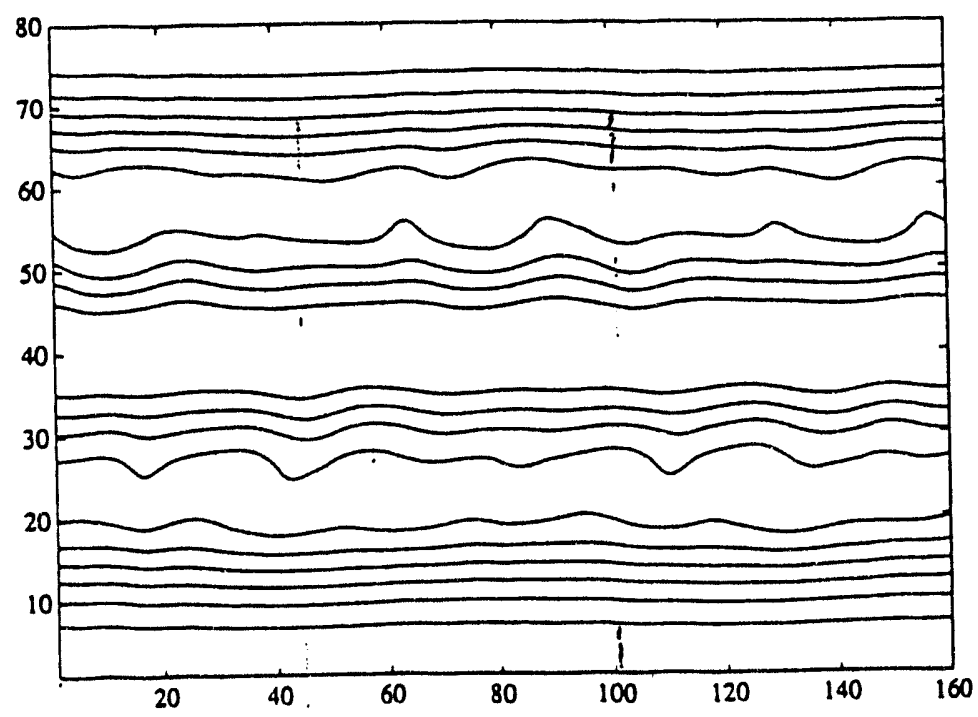

$F_{i} e_{i} r e / i$
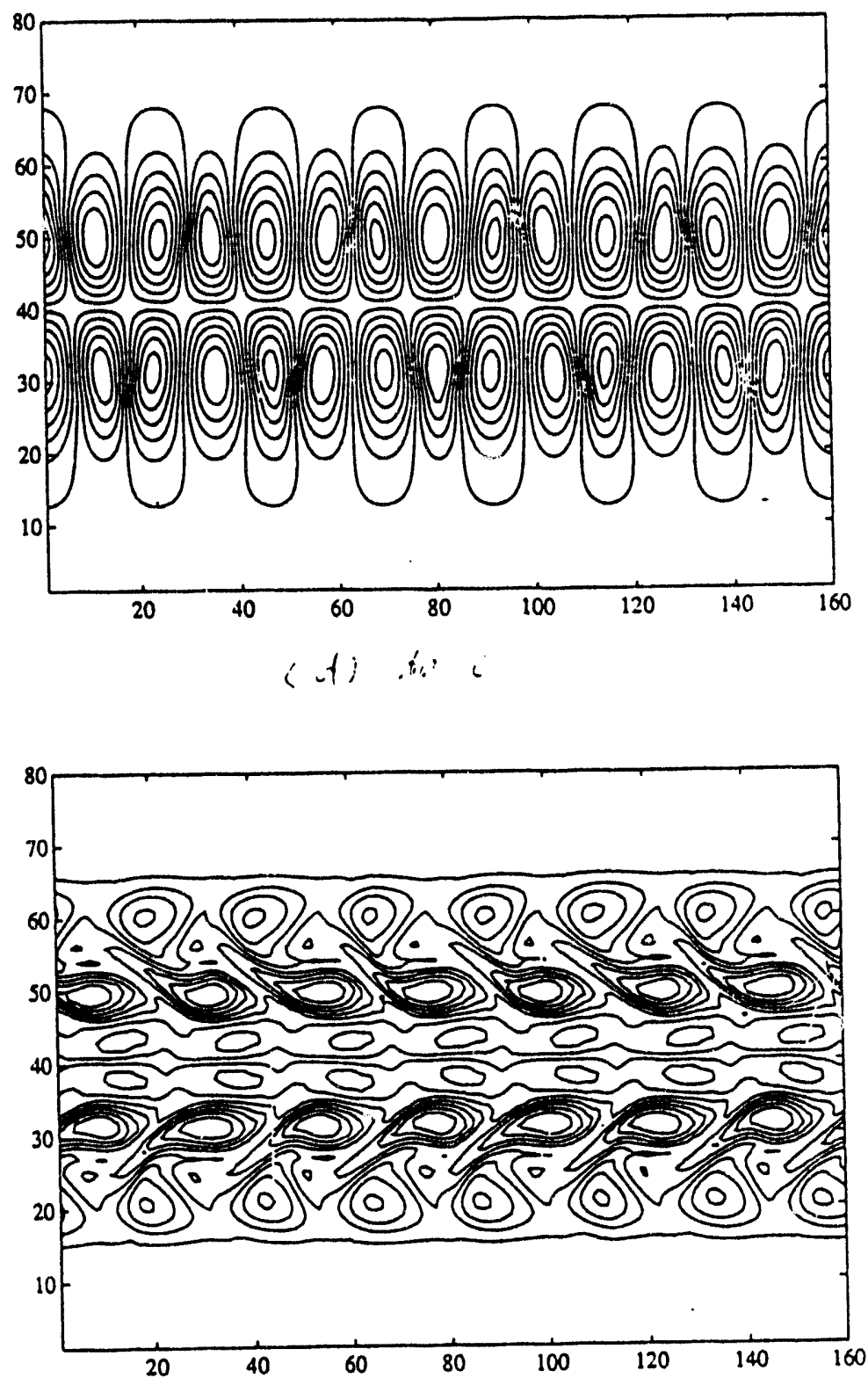

$$
\because 0 \text { :4?, }
$$
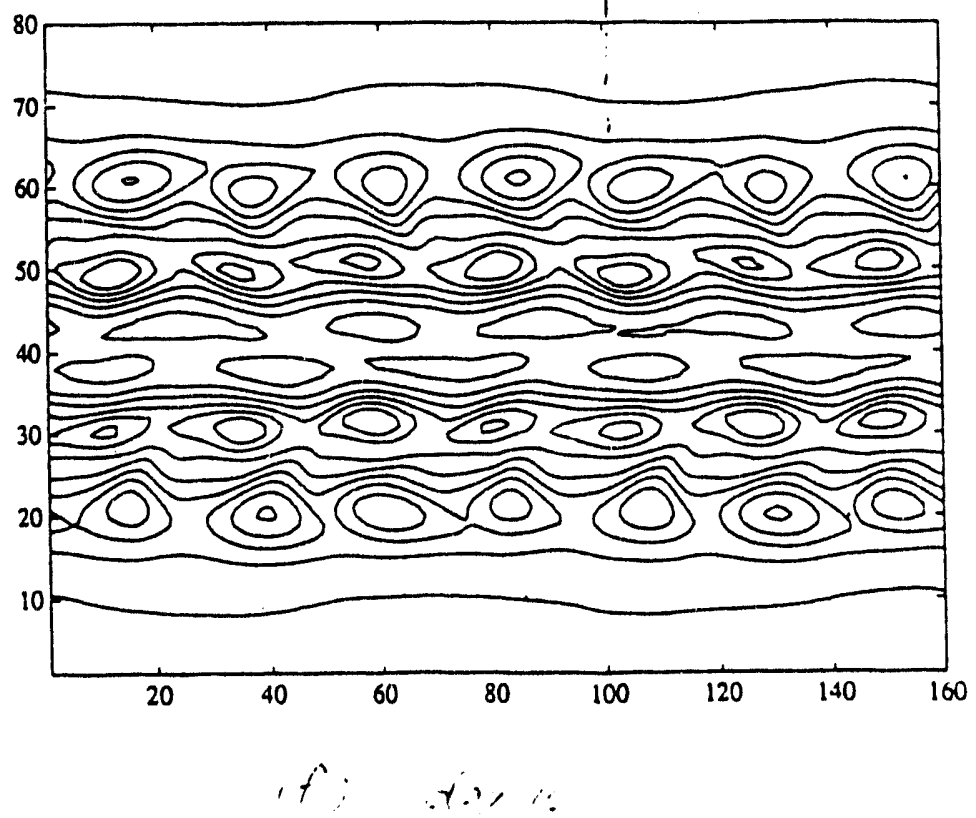

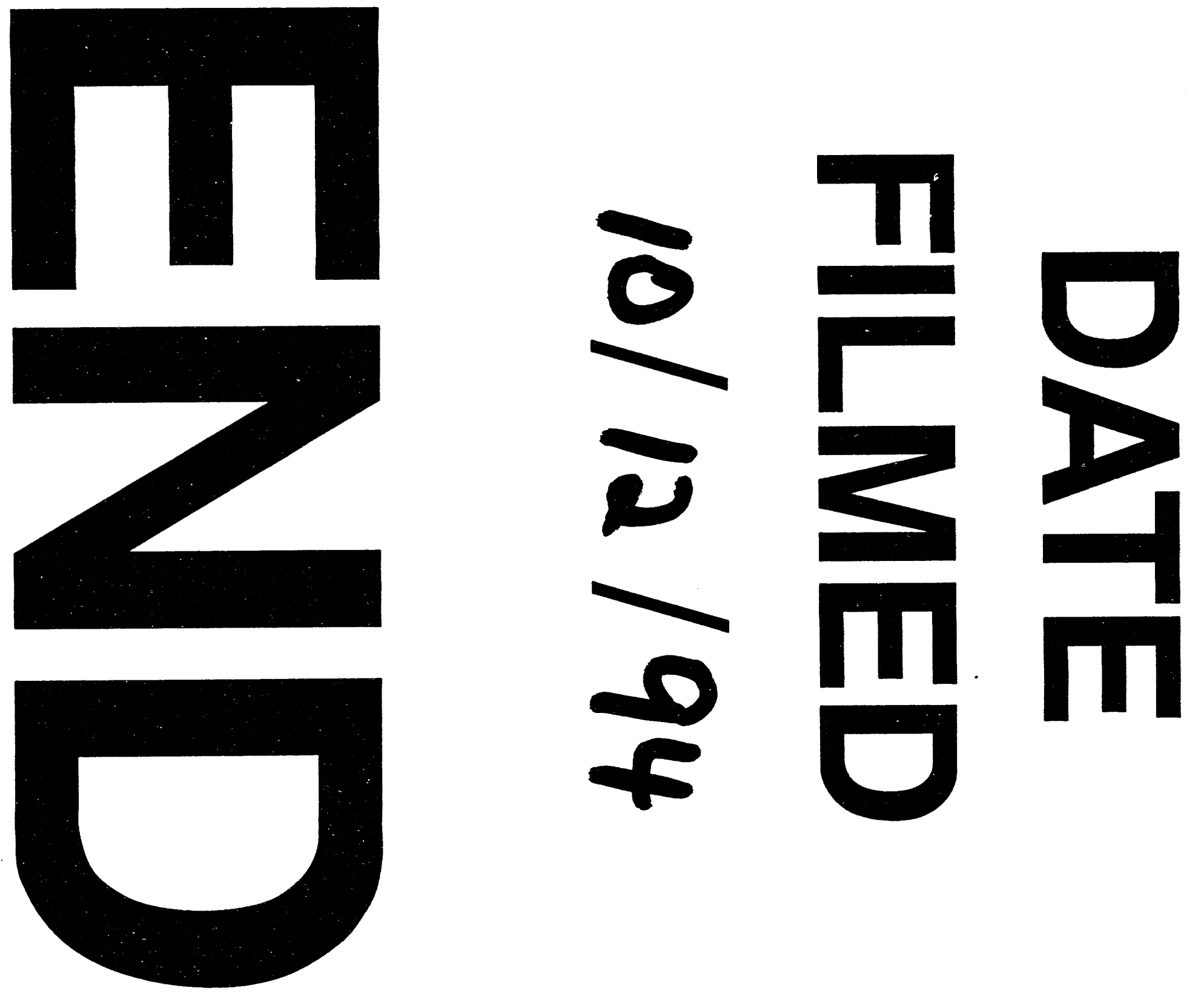


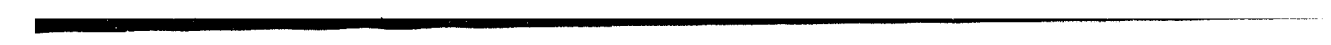

1

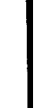

